\title{
Tracer diffusion in small periodic systems
}

\author{
L. F. Perondi and P.-M. Binder \\ Department of Physics, Theoretical Physics, University of Oxford, 1 Keble Road, Oxford OX1 3NP, United Kingdom
}

(Received 23 November 1992; revised manuscript received 8 February 1993)

\begin{abstract}
We study tracer diffusion in lattices consisting of identical replicas of smaller systems. We develop an exact enumeration method for computing the diffusion coefficient, which involves mapping the system into a single-particle random walk in a different geometry in configuration space. In one dimension the simulations show a crossover to normal diffusion, in agreement with mode-coupling results. In two dimensions the diffusion coefficient is enhanced by finite-size effects: we show this for a $3 \times 3$ periodic system. We find exact solutions for the single-vacancy case; our simulations agree with theory essentially to machine precision. We also develop an approximate theory for the periodic system for arbitrary concentrations of background particles, in close agreement with the numerical results.
\end{abstract}

\section{INTRODUCTION}

The simple hopping motion of classical particles has been used to describe a wide variety of physical phenomena: ionic motion in superionic conductors, ${ }^{1}$ atomic diffusion in hot solids via the vacancy mechanism, ${ }^{2}$ and diffusion of hydrogen in various metal hydrides. ${ }^{3}$ The usual simplifications in formulating hopping models consist of placing the particles at the nodes of a regular lattice, and imposing hard-core interactions in the form of an exclusion principle, forbidding a particle from hopping to an already occupied site. In this context, considering the properties of a single tagged random walker is known as the tracer problem.

The tracer problem is far from trivial: it was realized quite early ${ }^{4}$ that steps far away in time can be correlated. Exact solutions are only known in the limits of vanishingly small concentrations of background particles or vacancies. ${ }^{5}$ The self-avoidance gives rise to two effects on the motion of the tagged particle which substantially differentiate it from a free-particle random walk. First, a blocking effect which decreases the number of successful hops of the tagged particle, leading to a decrease in the value of the mean-squared displacement (MSD), and hence of the diffusion coefficient. Second, the walk performed by the tracer particle is correlated: the probability that there are two successive jumps in opposite directions is significantly higher than that of two successive jumps in the same direction, as the tracer leaves a vacancy behind. This effect is accentuated at higher concentrations of background particles. In addition to the two exactly solved limits, there are a variety of formal schemes yielding approximate solutions for intermediate concentrations. ${ }^{6-8}$

A number of Monte Carlo (MC) studies of tracer diffusion have also been performed. ${ }^{8,9}$ The numerical measurements agree with the exact and approximate theories typically within 1-2\%. In this paper we consider tracer diffusion in the case of a small repeated system of background particles (or periodic boundary conditions, but taking into account the tracer's crossing of system boundaries) for the following reasons. Firstly, we have developed an exact enumeration method to simulate this specific problem which is free of statistical error, unlike standard MC simulations. Secondly, this method allows us to see clearly and dramatically the effect of finite size in simulations, as we concentrate on extremely small systems $(3 \times 3)$. Finally, as we are able to develop exact solutions for the single-vacancy case and approximate solutions for arbitrary concentrations, we are able to report remarkable agreement between theory and simulations in those cases, and to observe how the finite-size effects vary in the single-vacancy case in particular.

The paper proceeds as follows. In Sec. II we develop the exact enumeration technique. Section III contains analytical results for the single-vacancy case and approximate results for general densities, as well as numerical results which are compared with theory. Finally, we discuss the results in Sec. IV.

\section{EXACT ENUMERATION METHOD}

The exact enumeration technique has been applied in a variety of random walk and cellular automata problems since its introduction in 1984 by Havlin and co-workers. ${ }^{10}$ It consists in evolving not individual particles or walkers but rather probabilities or distributions, according to the same or equivalent dynamical rules. Its advantage is that, for any configuration of fixed disorder (if applicable), the results are exact. The only averaging required is over different such configurations. ${ }^{11}$ The original applications concerned survival times in environments with randomly placed traps; subsequent applications have measured first passage times, velocity correlation functions, and other quantities of interest. Most applications have required only minor modifications of the original technique. These have included lattice gas cellular automata, ${ }^{12}$ in which particles interact, but can coexist at a lattice node, and Lorentz models ${ }^{13}$ in which particles interact with hardcore fixed obstacles. The application of the technique to 
the tracer problem is less straightforward.

We consider a finite system with $N_{S}$ sites, $N_{P}$ background particles, and a tagged particle. The motion of the tagged particle in the finite system can be thought of as motion in an infinite system if the latter is formed by repeating in space the original finite system. This artifice is usual in standard Monte Carlo simulations. We now consider the configuration space of such an infinite system. Let us define neighboring states as those in which the position of one particle differs exactly by one. A lattice with a complex topology is formed by linking neighboring states. If the position of the tagged particle is used as the reference for the position of the other particles, it is possible to arrange the lattice in configuration space in a regular way, which resembles the real-space lattice. The resulting lattice is formed by exact replicas of a basic unit, which is derived from the possible configurations that the system can assume with the tagged particle fixed at a given position in the real lattice. Figure 1 illustrates this phase-space arrangement for a one-dimensional system with $N_{S}=5$ and $N_{P}=2$. The evolution of the system in real space can be mapped into a single "particle" random walk in the configuration space. Due to the topological equivalence between the sets of states available to the system for different positions of the tracer in the real-space lattice, a general random walk in phase space is equivalent to a closed walk involving only the
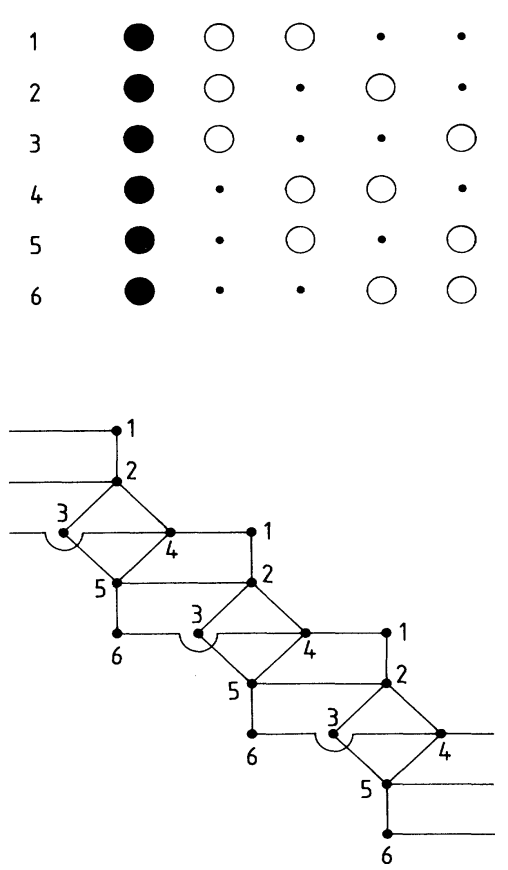

FIG. 1. Example of the mapping from real space to configuration space. There are six possible configurations of the tracer at the origin plus two background particles in a total of five sites. The geometry for the corresponding walk in configuration space is given in the lower part of the figure. The system moves from one set of six states to its neighboring set on the left or right whenever the tracer particle moves left or right. states corresponding to one of the building blocks just described. Figure 2 illustrates the situation for the example in Fig. 1.

The closed random walk can be described in terms of a finite transition matrix $\mathbf{T}$. In order to keep track of the position of the tracer while the point system performs a given path in the closed random walk, we associate a factor $e^{i \theta_{j}}\left(e^{-i \theta_{j}}\right)$ to each transition which involves a positive (negative) displacement of the tracer in a direction $j$ in real space. For a one-dimensional system, the probability of a displacement $l$ of the tracer during which the system goes from state $m$ to state $n$ in $N$ steps is given by

$$
P_{l}^{m n}(N)=\frac{1}{2 \pi} \int_{-\pi}^{\pi}\left(\mathbf{T}^{N}\right)_{m n} e^{-i l \theta} d \theta .
$$

This method of recording displacement is based on the fact that a path is constituted by a succession of transitions and that the probability associated with it is given by the product of the probabilities associated with each individual transition. It can be seen as a direct extension of the formalism given, for example, by $\mathrm{Ziman}^{14}$ for the treatment of an unrestricted one-particle random walk. Equation (1) can be easily generalized to higher dimensions.

In order to further illustrate the method we develop in full the one-dimensional example introduced above, by constructing explicitly its closed-walk transition matrix $\mathbf{T}$. In the following we denote the lattice coordination number by $Z$ and the total number of particles by $N_{T} \equiv N_{P}+1$. A point to be noted is that, in a standard $\mathrm{MC}$ run, the total number of transitions that a system can undergo in any of its possible states is $N_{T} Z$. A fraction of these are transitions to the same configurationthey correspond to those instances in a $\mathrm{MC}$ simulation in which the configuration is left unchanged in a system step. From Figs. 1 and 2 it can be seen that the diagonal elements corresponding to states 1,3 , and 6 have value $4 / 6$, while for states 2,4 , and 6 , they are $2 / 6$. The transitions $1 \rightarrow 4,4 \rightarrow 6$, and $2 \rightarrow 5$ are caused by a

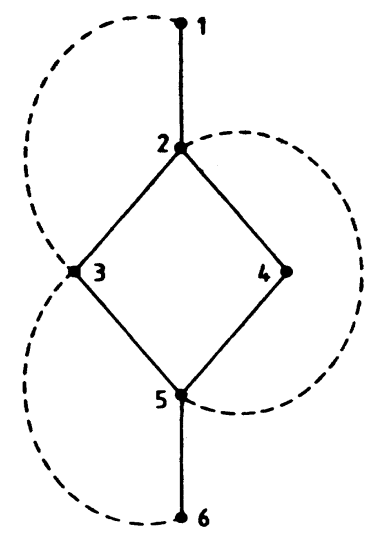

FIG. 2. Single-system (closed) random walk for the example of Fig. 1. 
tracer jump to the left, and have a factor $e^{-i \theta}$ associated with them. The respective reverse transitions, caused by a tracer jump to the right, have a factor $e^{i \theta}$ in the transition matrix. Transitions involving jumps of background particles have value $1 / Z N_{T}$. The full transition matrix for the system illustrated in Figs. 1 and 2 is given by

$$
\mathbf{T}=\frac{1}{6}\left(\begin{array}{cccccc}
4 & 1 & 0 & e^{-i \theta} & 0 & 0 \\
1 & 2 & 1 & 1 & e^{-i \theta} & 0 \\
0 & 1 & 4 & 0 & 1 & 0 \\
e^{i \theta} & 1 & 0 & 2 & 1 & e^{-i \theta} \\
0 & e^{i \theta} & 1 & 1 & 2 & 1 \\
0 & 0 & 0 & e^{i \theta} & 1 & 4
\end{array}\right)
$$

In what follows we develop in general terms the formulas which lead to the tracer mean-squared displacement (MSD) in the case of a one-dimensional system.

The probability $P_{l}(N)$ that the tracer is at position $l$ after $N$ time steps, averaged over all possible initial conditions, is given by

$$
P_{l}(N)=\frac{1}{2 \pi M} \int_{-\pi}^{\pi} \sum_{i j}\left(\mathbf{T}^{N}\right)_{i j} e^{-i l \theta} d \theta,
$$

where $M=\left(\begin{array}{c}N_{S}-1 \\ N_{P}\end{array}\right)$ is the number of possible states of the system. $P_{l}$ in turn is related to $\mathbf{T}$ by the inversion formula

$$
\sum_{l} P_{l}(N) e^{i \theta l}=\frac{1}{M} \sum_{i j}\left(\mathbf{T}^{N}\right)_{i j}
$$

The expectation value of the MSD after $N$ time steps can be obtained by differentiating twice the above expression:

$$
\sum_{l} l^{2} P_{l}(N)=\left.\frac{1}{M}\left[-\frac{\partial^{2}}{\partial^{2} \theta}\left(\sum_{i j} \mathbf{T}^{N}\right)_{i j}\right]\right|_{\theta=0} .
$$

We now show a simple way to evaluate the second derivative in the previous expression. We expand the transition matrix $\mathbf{T}$ in powers of $\theta$ :

$$
\mathbf{T}(\theta)=\mathbf{A}+i \theta \mathbf{B}-\frac{\theta^{2}}{2} \mathbf{C}+\mathcal{O}\left(\theta^{3}\right) .
$$

Squaring the above expression to the same order one gets

$$
\begin{aligned}
\mathbf{T}^{2}(\theta) & =\left(\mathbf{A}+i \theta \mathbf{B}-\frac{\theta^{2}}{2} \mathbf{C}\right)\left(\mathbf{A}+i \theta \mathbf{B}-\frac{\theta^{2}}{2} \mathbf{C}\right) \\
& =\mathbf{A}^{2}+i \theta(\mathbf{A B}+\mathbf{B A})-\frac{\theta^{2}}{2}\left(\mathbf{A} \mathbf{C}+\mathbf{C A}+\mathbf{2 B}^{2}\right) \\
& =\mathbf{A}_{2}+i \theta \mathbf{B}_{2}-\frac{\theta^{2}}{2} \mathbf{C}_{2}
\end{aligned}
$$

and in general

$$
\begin{aligned}
\mathbf{T}^{n}(\theta)= & \mathbf{A}_{n-1} \mathbf{A}+i \theta\left(\mathbf{B}_{n-1} \mathbf{A}+\mathbf{A}_{n-1} \mathbf{B}\right) \\
& -\frac{\theta^{2}}{2}\left(\mathbf{A}_{n-1} \mathbf{C}+\mathbf{C}_{n-1} \mathbf{A}+2 \mathbf{B}_{n-1} \mathbf{B}\right) \\
= & \mathbf{A}_{n}+i \theta \mathbf{B}_{n}-\frac{\theta^{2}}{2} \mathbf{C}_{n}
\end{aligned}
$$

where

$$
\begin{aligned}
& \mathbf{A}_{n}=\mathbf{A}_{n-1} \mathbf{A}, \\
& \mathbf{B}_{n}=\mathbf{B}_{n-1} \mathbf{A}+\mathbf{A}_{n-1} \mathbf{B},
\end{aligned}
$$

and

$$
\mathbf{C}_{n}=\mathbf{A}_{n-1} \mathbf{C}+\mathbf{C}_{n-1} \mathbf{A}+2 \mathbf{B}_{n-1} \mathbf{B} .
$$

From (6) we have

$$
-\left.\frac{\partial^{2}}{\partial^{2} \theta} \sum_{i j}\left(\mathbf{T}^{N}\right)_{i j}(\theta)\right|_{\theta=0}=\sum_{i j}\left(\mathbf{C}_{N}\right)_{i j},
$$

and from (3) we have

$$
\left\langle R^{2}\right\rangle_{N}=\frac{1}{M} \sum_{i j}\left(\mathbf{C}_{N}\right)_{i j} .
$$

We use the above equation to compute the MSD; Eqs. (8)-(10) give a recursion relation to obtain $\mathbf{C}_{N}$.

The connection with standard MC simulations is that each power of the transition matrix corresponds to a jump trial by one particle, and a full MC step per particle corresponds to $N_{T}$ iterations of the matrix.

Finally, the diffusion constant $D$ is defined by

$$
\left\langle R^{2}\right\rangle_{N} \equiv D\left(\frac{N}{N_{P}}\right)
$$

the correlation factor $f$ is related to $D$ through $D=f f_{B}$. The blocking factor $f_{B}$ is the fraction of successful hops of the tagged particle out of a given number of attempted hops. By considering the probabilities of the possible environments that the tagged particle can experience, and the probabilities of a successful hop in each environment, we find

$$
f_{B}=\left(1-\frac{N_{P}}{N_{S}-1}\right)
$$

where $N_{P}$ is the number of background particles and $N_{S}$ is the number of sites in the system. This expression holds in higher dimensions. The blocking factor corresponding to an infinite system with a concentration $c$ of background particles $c$ is given by $(1-c)$. By comparing these two expressions we can relate the number of particles in the finite system and the concentration of background particles in the corresponding infinite system.

The procedure in two dimensions is similar, but the matrix has to take into account all transitions between configurations in which the tracer is at the $(0,0)$ position. The equation analogous to (6) is derived in the Appendix.

Finally, we remark that the time and space Fourier transforms of the tracer's self-correlation function can be calculated under the formalism of this section. These quantities are relevant to the interpretation of data related to spectroscopic techniques, such as Mössbauer spectroscopy and NMR signals of moving atoms. ${ }^{15}$

\section{RESULTS}

The method has proved to be quite fast, ${ }^{16}$ but also very memory consuming. The order of the matrix, as seen 
before, is $\left(\begin{array}{c}N_{S}-1 \\ N_{P}\end{array}\right)$, which grows essentially exponentially with $N_{S}$ for particle concentrations of $\sim 1 / 2$. Therefore, the method is most appropriate for very small lattices, or in the limits of low density of background particles or vacancies.

After showing an example in one dimension, we concentrate on $3 \times 3$ lattices as well as the single-vacancy limit for larger two-dimensional lattices. Note that for the single-vacancy case, the order of the matrix $\mathbf{C}$ grows only linearly with system size; this allows us to consider systems up to $8 \times 8$. On the other hand, for a $4 \times 4$ system with 7 or 8 particles the order of the matrix is 6435 , beyond our present memory possibilities. The typical times needed to observe asymptotic behavior are about 500 full MC steps, or about 3000 jump trials for a 6-particle system.

Figure 3 shows the full evolution of the MSD vs time, as calculated by the matrix multiplication method presented in the previous section. The system is a onedimensional periodic array of 10 sites, with a tracer plus four background particles. It is clear that the time evolution of the MSD is extremely smooth when compared with standard MC simulations (see Refs. 8, 9 and 17). This is because our method averages over all possible evolutions of the system, rather than a few.

Secondly, notice that the final behavior is $\left\langle R^{2}\right\rangle \sim$ $t$, corresponding to normal diffusion. This is in contrast with the expected $t^{1 / 2}$ abnormal asymptote in one dimension. ${ }^{18,19}$ Note also a crossover from a $t^{1 / 2}$-like behavior to the final linear behavior. This fact can be understood in terms of finite-size effects as follows. In the single-vacancy limit, the expression for the average cosine of the angle between successive jumps of the tagged particle can be generally expressed as (see Appendix)

$\cos \theta=\frac{-1}{2 d L_{1} L_{2}} \sum_{k_{1}=1}^{L_{1}-1} \sum_{k_{2}=0}^{L_{2}-1} \frac{1-e^{-i 4 \pi k_{1} / L_{1}}}{1-\frac{1}{2}\left(\cos \frac{2 \pi k_{1}}{L_{1}}+\cos \frac{2 \pi k_{2}}{L_{2}}\right)}$,

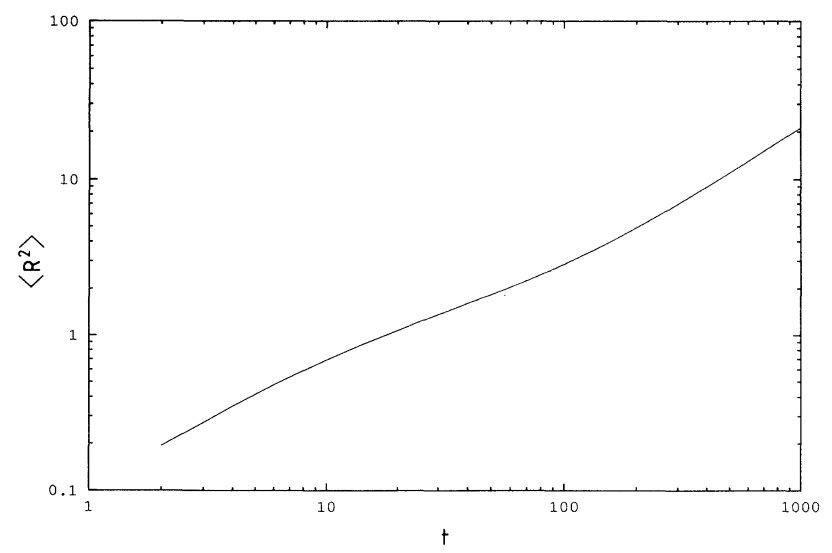

FIG. 3. Mean-squared displacement vs time (logarithmic plot) for one-dimensional tracer diffusion; 10 sites, 4 background particles. The final behavior is $\left\langle R^{2}\right\rangle \sim t$, crossing over from an apparent $\left\langle R^{2}\right\rangle \sim t^{1 / 2}$ at times of order 50 . where $d=1,2$ is the space dimension and $L_{1}$ and $L_{2}$ denote the lattice dimensions. In the one-dimensional case $L_{1}=N_{s}, L_{2}=1$, and all references to the sum over $k_{2}$ should be dropped. In this circumstance $|\cos \theta|<1$ for finite $N_{s}$ and the diffusion coefficient is given by

$$
D=f_{B} \frac{1+\cos \theta}{1-\cos \theta} \text {. }
$$

Hence in the single-vacancy limit, normal diffusion will be observed as a consequence of the periodic boundary conditions. In the limit $N_{s} \rightarrow \infty$, (15) shows that $\cos \theta \rightarrow-1$ and the normal diffusive behavior disappears. The presence of other vacancies in the lattice changes the general form of the correlation between displacements, leading to a less correlated random walk for the tracer. In this situation, normal diffusion can also be expected for long enough times. For short times, on the other hand, the periodic boundary conditions have practically no influence on the tracer's motion, and we expect the MSD to have the same time dependence as in the infinite system. The crossover observed in Fig. 3 is therefore purely a finite-size effect. A fair estimate of the crossover time is given by the characteristic time needed for a vacancy to return to its original position by crossing one of the two boundaries of the finite lattice.

Figure 4 shows the correlation factor for tracer diffusion in a $3 \times 3$ periodic lattice (crosses), compared with large-lattice results (squares) obtained by Tahir-Kheli and El-Meshad. ${ }^{8}$ We have computed the MSD for the equivalent of about $600 \mathrm{MC}$ steps per particle, and our results for $\left\langle R^{2}\right\rangle / N$ have converged to at least 8 significant figures. The observed enhancement of the diffusion coefficient, of up to $25 \%$ for high concentrations, is again a finite-size effect. Equation (15) now with $L_{1} \times L_{2}=N_{s}$ and $d=2$ shows that $\cos \theta$ tends to the limit $-1+2 / \pi$ (see Ref. 17) as $N_{s} \rightarrow \infty$. Hence Eq. (16) is always valid and the diffusive behavior observed in two dimensions is not an accident due to the periodic boundary conditions, but rather an intrinsic characteristic of the system. The presence of the periodic boundary conditions, however,

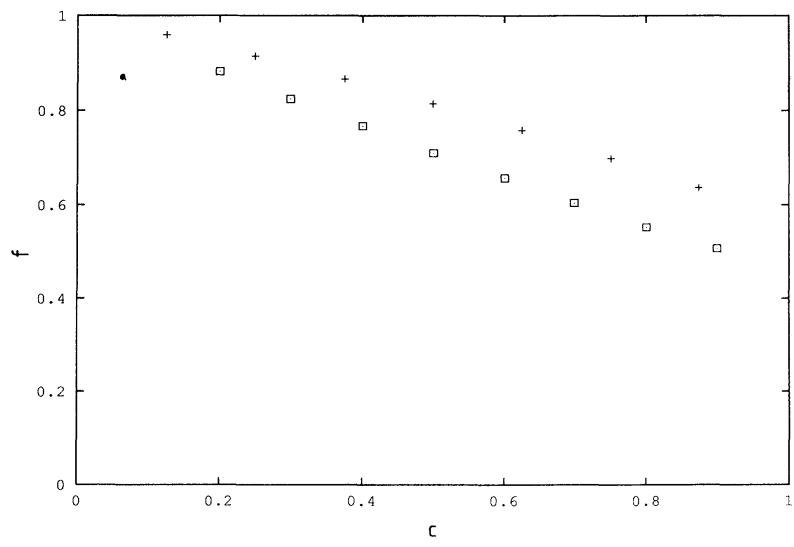

FIG. 4. Structure factor $f$ vs particle concentration $c$. Open squares: Tahir-Kheli-El-Meshad simulations (Ref. 8); crosses: exact enumeration results in a $3 \times 3$ lattice, illustrating the effects of finite size in simulation. 
enhances the value of the diffusion coefficient: The value of $\cos \theta$ given by (15) for finite $N_{s}$ is always larger than the value for the infinite lattice. Hence, the diffusion coefficient will decrease in magnitude for increasing $N_{S}$, tending asymptotically to

$$
D=f_{B} \frac{1}{\pi-1}
$$

for $N_{S} \rightarrow \infty$.

Numerical results from the method of Sec. II are compared against the exact result above in Table I. The results for the correlation factor agree essentially to numerical precision up to the system sizes $(8 \times 8)$ tested. For completeness, we also have included an approximate expression for the single-vacancy case and finite systems, recently obtained by Brummelhuis and Hilhorst. ${ }^{20}$ This is to our knowledge the only other result for tracer diffusion which applies specifically to finite lattices. Since their result is obtained only to order $1 / L^{2}$, it is no surprise that our simulations agree to a greater extent with (15) in this paper.

As a final point we compare our $3 \times 3$ periodic lattice results with an adaptation of the TKE method for finite lattices. Starting from a mean-field decoupling approximation for many-particle rate equations, Tahir-Kheli and Elliott $\left(\mathrm{TKE}^{7}\right)$ obtained an approximate expression for the tracer diffusion coefficient for arbitrary concentrations of background particles in the infinite lattice. They obtained

$$
D=f_{B}\left(1-\frac{2 c \cos \theta}{(2-c)(1+\cos \theta)}\right)^{-1}
$$

This expression is exact in the limits of a vanishingly small concentrations of background particles or vacancies.

In order to adapt the TKE result to finite periodic lattices, one must replace $\cos \theta$ by the finite-lattice result, as given in (15). In addition, the limiting cases of a single vacancy or a single background particle now correspond respectively to concentrations $\left(N_{S}-2\right) /\left(N_{S}-1\right) \neq 1$ and $1 /\left(N_{S}-1\right) \neq 0$. Taking these limits into account we obtain

$$
f=1+\frac{c\left(N_{S}-1\right) /\left(N_{S}-2\right)}{\left(\frac{1+r}{2}\right)\left(\frac{1+\cos \theta}{\cos \theta}\right)-s}
$$

where $r=\left[N_{S}-2-\left(N_{S}-1\right) c\right] /\left(N_{S}-3\right)$ and $s=\left[\left(N_{S}-\right.\right.$ 1) $c-1] /\left(N_{S}-3\right)$.

The correlation factor results for the $3 \times 3$ lattice shown in Fig. 4 are presented in tabular form, and compared with the adapted TKE theory in Table II. In this case, as the number of microscopic time steps required for asymptotic behavior is reasonably small (1200-4800 steps), we do not have to worry much about roundoff or convergence problems. The numerical and analytical results agree to within $\sim 0.5 \%$.

\section{SUMMARY AND DISCUSSION}

In this paper we have developed several advances in the understanding of the tracer problem, particularly to the case of small periodic systems. In Sec. II we developed an exact enumeration method for calculating numerically the diffusion coefficient of a tracer particle. This method has no statistical errors - the only errors are due to roundoff, or to iterating for finite times. The advantages of the method are seen in particular in Table I, which shows the correlation factor for the single-vacancy case, obtained both exactly and by simulations (exact enumeration method). The relative error is, to our knowledge, several orders of magnitude lower than obtained in any previous simulation of this problem. Thanks to the new method we confirm crossover behavior to $\left\langle R^{2}\right\rangle \sim t$ in one dimension for periodic systems (Sec. III), which was predicted by mode-coupling theory ${ }^{17}$ and by the analytical results of this paper. In Sec. III we also show clearly the enhancement of the diffusion coefficient caused by finite-size effects in two dimensions. We have also obtained exact results for the single-vacancy case, which improved on a previous approximate result. ${ }^{20}$ Our expression is confirmed by our new simulational method. Finally, we modify an approximate theory ${ }^{7}$ for arbitrary concentrations of background particles, to take into account finite-system size. The results, presented in Table II, are in good agreement with simulations. As in the infinite lattice, the modified TKE theory tends to overestimate the value of the diffusion coefficient at intermediate particle concentrations.

TABLE I. Single-vacancy results for an $L \times L$ lattice: size $L$, and correlation factor from $1 / L^{2}$ expansion (Brummelhuis and Hilhorst, Ref. 20), numerical (exact enumeration) method, and exact result [Eqs. (15) and (16)]. Relative error refers to the exact enumeration method compared to the exact result. The value in the last column is the best fit of $a$ for $\left\langle R^{2}\right\rangle=a+D N$.

\begin{tabular}{llcccc}
\hline \hline$L$ & $f(\mathrm{BH})$ & $f$ (numerical) & $f$ (exact) & Relative error & $a$ (constant) \\
\hline 3 & 0.6384 & 0.63636367 & 0.63636364 & $5.4 \times 10^{-8}$ & 0.037187 \\
4 & 0.5634 & 0.54838750 & 0.54838710 & $7.3 \times 10^{-7}$ & 0.032189 \\
5 & 0.5287 & 0.51515151 & 0.51515151 & $-1.2 \times 10^{-8}$ & 0.026018 \\
6 & 0.5098 & 0.49910767 & 0.49910767 & $1.2 \times 10^{-9}$ & 0.021158 \\
8 & 0.4910 & 0.48440758 & 0.48440748 & $2 \times 10^{-7}$ & 0.014697 \\
\hline \hline
\end{tabular}


TABLE II. Comparison of exact enumeration results and adapted Tahir-Kheli-Elliott (TKE) theory for $3 \times 3$ lattice. $N_{P}$ is the number of background particles in addition to the tracer. Numbers in the fourth column are obtained by standard Monte Carlo simulation for the same systems (50000 independent particle histories).

\begin{tabular}{cccc}
\hline \hline$N_{P}$ & $f$ (enumeration) & $f(\mathrm{TKE})$ & $f$ (Monte Carlo) \\
\hline 1 & 0.9592 & 0.9592 & 0.9474 \\
2 & 0.9146 & 0.9153 & 0.9182 \\
3 & 0.8662 & 0.8681 & 0.8614 \\
4 & 0.8140 & 0.8171 & 0.8151 \\
5 & 0.7581 & 0.7619 & 0.7533 \\
6 & 0.6988 & 0.7019 & 0.7022 \\
7 & 0.6364 & 0.6364 & 0.6446 \\
\hline \hline
\end{tabular}

\section{ACKNOWLEDGMENTS}

The authors thank the Science and Engineering Research Council (U.K.) for support. L.F.P. thanks the Brazilian Research Council (CNPq) and the Brazilian Space Research Institute (INPE) for financial support. The authors also acknowledge useful discussions with Professor R. J. Elliott.

\section{APPENDIX}

\section{Angle expansion in two dimensions}

The double Fourier transform and inversion formula for the two-dimensional system analogous to (1) and (2) are

$$
P_{l_{1}, l_{2}}(N)=\frac{1}{(2 \pi)^{2} M} \iint \sum_{i j}\left(\mathbf{T}^{N}\right)_{i j} e^{-i \theta_{1} l_{1}-i \theta_{2} l_{2}} d \theta_{1} d \theta_{2}
$$

and

$$
\sum_{l_{1}} \sum_{l_{2}} P_{l_{1}, l_{2}}(N) e^{i \boldsymbol{\theta} \cdot \boldsymbol{l}}=\frac{1}{M} \sum_{i j}\left(\mathbf{T}^{N}\right)_{i j},
$$

respectively. The MSD is then given by

$$
\begin{aligned}
\left\langle R^{2}\right\rangle_{N} & =\sum_{l_{1}} \sum_{l_{2}}\left(l_{1}^{2}+l_{2}^{2}\right) P_{l_{1}, l_{2}}(N) \\
& =\left.\left[\left(-\frac{\partial^{2}}{\partial^{2} \theta_{1}}-\frac{\partial^{2}}{\partial^{2} \theta_{2}}\right) \frac{1}{M} \sum_{i j}\left(\mathbf{T}^{N}\right)_{i j}\right]\right|_{\theta=0} .
\end{aligned}
$$

The angle expansion analogous to (4) is now

$$
\mathbf{T}\left(\theta_{1}, \theta_{2}\right)=\mathbf{A}+i \theta_{1} \mathbf{B}_{x}-\frac{\theta_{1}^{2}}{2} \mathbf{C}_{x}+i \theta_{2} \mathbf{B}_{y}-\frac{\theta_{2}^{2}}{2} \mathbf{C}_{y}
$$

Note that mixed products do not contribute to $\left\langle R^{2}\right\rangle_{N}$.
Because of symmetry

$$
\left.\frac{\partial^{2}}{\partial^{2} \theta_{1}} \frac{1}{M} \sum_{i j}\left(\mathbf{T}^{N}\right)_{i j}\right|_{\theta=0}=\left.\frac{\partial^{2}}{\partial^{2} \theta_{2}} \frac{1}{M} \sum_{i j}\left(\mathbf{T}^{N}\right)_{i j}\right|_{\theta=0}
$$

and we only have to consider powers of

$$
\mathbf{A}+i \theta_{1} \mathbf{B}_{x}-\frac{\theta_{1}^{2}}{2} \mathbf{C}_{x}
$$

This is the equivalent of (6) in two dimensions. The rest of the derivation proceeds as shown in Sec. II.

\section{Derivation of Eq. (15)}

The tracer's average MSD after a large number of time steps $N$ can be expressed as

$$
\begin{aligned}
R^{2} & =\sum_{i=1}^{N^{\prime}} \sum_{j=1}^{N^{\prime}} \overline{\mathbf{r}_{\mathbf{i}} \cdot \mathbf{r}_{\mathbf{j}}} \\
& \approx f_{B} N\left(1+2 \sum_{l} \cos \theta_{l}\right),
\end{aligned}
$$

where $f_{B}$ is the blocking factor, $N^{\prime}=f_{B} N$ is the number of successful tracer steps, and $\cos \theta_{l}$ is the average cosine of the angle between displacements separated by $l$ successful steps. These steps refer to the infinite lattice. Equation (A8) is valid and asymptotically correct in the limit $N \rightarrow \infty$ provided the sum $\sum_{l} l \cos \theta_{l}$ is convergent. In the single-vacancy limit, displacements arbitrarily far apart are correlated, and it can be shown that

$$
\cos \theta_{l}=(\cos \theta)^{l},
$$

where $\cos \theta$ designates the average cosine of the angle between successive jumps. In order to evaluate $\cos \theta$ we have to consider the random walk performed by the single vacancy in the periodic lattice. Consider the situation in which the tracer and vacancy have just exchanged positions, and let $r$ and $i$ designate the positions of the tracer and vacancy, respectively. Let $m$ designate the other site neighboring $r$. Then,

$$
\cos \theta=P_{m i}-P_{i i},
$$

where $P_{m i}, P_{i i}$ are the probabilities that the vacancy returns to site $r$ for the first time through site $m$ or through site $i$, respectively. These probabilities can be expressed in terms of generating functions; they satisfy the difference equation

$$
U_{l j}(\lambda)=\delta_{l j}+\lambda \sum_{s} p_{l s} U_{s j}(\lambda),
$$

with $U$ defined by

$$
U_{l j}(\lambda)=\sum_{n=0}^{\infty} \lambda^{n} P_{l j}(N),
$$

where $P_{l j}(N)$ is the probability that a random walker 
initially occupying site $j$ is at site $l$ after $N$ steps. In the expressions above, $p_{l s}$ is the probability of a transition between sites $l$ and $s$. In terms of these functions (see Sholl ${ }^{15}$ ),

$$
P_{l i}=\frac{1}{2}\left(U_{l i}(1)-\frac{U_{l r}(1) U_{r i}(1)}{U_{r r}(1)}\right) \quad(l=i, m) .
$$

The above equations should in formal terms be viewed as a limiting process, since the generating functions are divergent for $\lambda=1$. Equation (A13) can be solved for
$U_{l j}(\lambda)$ by Fourier series, yielding

$\cos \theta=\frac{-1}{2 d L_{1} L_{2}} \sum_{k_{1}=0}^{L-1} \sum_{k_{2}=0}^{L-1} \frac{1-e^{-i 4 \pi k_{1} / L}}{1-\frac{1}{2}\left(\cos \frac{2 \pi k_{1}}{L}+\cos \frac{2 \pi k_{2}}{L}\right)}$

where $L_{1}, L_{2}$ are the size of the system and $d$ is the dimension. For $L_{1}=1$, the one-dimensional case, (A14) simplifies to a single summation.
${ }^{1}$ For a review, see Physics of Superionic Conductors, edited by M. B. Salamon (Springer, New York, 1979).

${ }^{2}$ For a review, see A. D. LeClaire, in Physical Chemistry, edited by W. Jost (Academic, New York, 1970), Vol. 10.

${ }^{3} \mathrm{~T}$. Springer, in Vol. 3 of Topics in Current Physics, edited by M. Lovesey and T. Springer (Springer, New York, 1977).

${ }^{4}$ J. Bardeen, Phys. Rev. 76, 1403 (1949); J. Bardeen and C. Herring, in Imperfections in Nearly Perfect Crystals, edited by W. Shockley (Wiley, New York, 1950), p. 261.

${ }^{5}$ R. A. Tahir-Kheli and R. J. Elliott, J. Phys. C 15, L445 (1982); 15, 5751 (1982).

${ }^{6}$ P. A. Fedders and O. F. Sankey, Phys. Rev. B 15, 3580 (1977); 18, 5938 (1978); O. F. Sankey and P. A. Fedders, ibid. 15, 3586 (1977); 20, 39 (1979); 22, 5135 (1980).

${ }^{7}$ R. A. Tahir-Kheli and R. J. Elliott, Phys. Rev. B 27, 844 (1983).

${ }^{8}$ R. A. Tahir-Kheli and N. El-Meshad, Phys. Rev. B 32, 6166 (1985).

${ }^{9}$ K. W. Kehr, R. Kutner, and K. Binder, Phys. Rev. B. 23, 4931 (1981).

${ }^{10} \mathrm{~S}$. Havlin, G. H. Weiss, J. E. Kiefer, and M. Dishon, J. Phys. A 17, L347 (1984).

${ }^{11}$ The present application of the technique does not require averaging over fixed disorder, as the background disorder is purely dynamical. In such cases, the results are exact. For another recent application of the technique which requires no disorder averaging, see P.-M. Binder, J. Stat. Phys. 67, 827 (1992).

${ }^{12}$ D. Frenkel and M. H. Ernst, Phys. Rev. Lett. 63, 2165 (1989); M. A. van der Hoef and D. Frenkel, Phys. Rev. A 41, 4277 (1990).

${ }^{13}$ D. Frenkel, F. van Luijn, and P.-M. Binder, Europhys. Lett. 20, 7 (1992).

${ }^{14} \mathrm{~J}$. M. Ziman, Models of Disorder (Cambridge University Press, Cambridge, United Kingdom, 1979), Sec. 7.8.

${ }^{15}$ H. C. Torrey, Phys. Rev. 92, 262 (1953); K. S. Singwi and A. Sjölander, ibid. 119, 863 (1960); C. J. Chudley and R. J. Elliott, Proc. Phys. Soc. London 77, 33 (1961); C. A. Sholl, J. Phys. C 14, 1479 (1981).

${ }^{16}$ The iteration of the $126 \times 126$ matrices corresponding to the tracer and 4 background particles in 10 lattice sites for 1000 microsteps (200 MC steps/particle) takes about 9600 $\mathrm{s}$ in a Sparc workstation.

${ }^{17}$ H. van Beijeren, K. W. Kehr, and R. Kutner, Phys. Rev. B 28, 5711 (1983).

${ }^{18}$ T. E. Harris, J. Appl. Prob. 2, 323 (1965); P. M. Richards, Phys. Rev. B 16, 1393 (1977).

${ }^{19}$ R. Arratia, Ann. Prob. 11, 706 (1983).

${ }^{20}$ M. J. A. M. Brummelhuis and H. J. Hilhorst, J. Stat. Phys. 53, 249 (1988). 